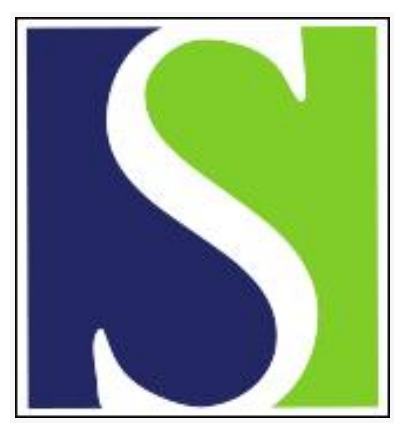

Scand J Work Environ Health 2005;31(5):360-366

https://doi.org/10.5271/sjweh.919

Issue date: Oct 2005

Acute effects of cigarette smoking on the heart rate variability of taxi drivers during work

by Kobayashi F, Watanabe T, Akamatsu Y, Furui H, Tomita T, Ohashi R, Hayano J

Affiliation: Department of Health and Psychosocial Medicine, Aichi Medical University School of Medicine, Aichi, Japan. fumio@aichi-med-u.ac.jp

Key terms: acute effect; autonomic nervous activity; cardiovascular disease; cigarette smoking; circadian rhythm; complex demodulation; heart rate variability; parasympathetic activity; sympathetic activity; taxi driver

This article in PubMed: www.ncbi.nlm.nih.gov/pubmed/16273962 


\title{
Acute effects of cigarette smoking on the heart rate variability of taxi drivers during work
}

\author{
by Fumio Kobayashi, MD, ${ }^{1}$ Takemasa Watanabe, MD, ${ }^{2}$ Yasuhiro Akamatsu, MD, ${ }^{1}$ Hikari Furui, MD, ${ }^{3}$ \\ Teruyuki Tomita, MD, ${ }^{1}$ Rumi Ohashi, PhD, ${ }^{4}$ Junichiro Hayano, MD ${ }^{5}$
}

Kobayashi F, Watanabe T, Akamatsu Y, Furui H, Tomita T, Ohashi R, Hayano J. Acute effects of cigarette smoking on the heart rate variability of taxi drivers during work. Scand J Work Environ Health 2005;31(5):360-366.

\begin{abstract}
Objectives This study attempted to clarify the acute effects of cigarette smoking on autonomic nervous function among taxi drivers under ordinary work conditions.

Methods Holter electrocardiographic recordings from 20 healthy middle-aged taxi drivers were analyzed for the time from 0800 in the morning to 0159 at night. The amplitudes (milliseconds) of the high-frequency (HF) component and the ratio of the low-frequency component to HF (LF/HF) were calculated as changes in the R-R interval, and time-course changes were investigated by a complex demodulation method. The exact starting time of smoking was identified with the use of a specially designed cigarette lighter. The mean LF/HF and HF for 5 minutes immediately prior to smoking were calculated as the baseline, and the means for every 5 minutes up to 15 minutes were calculated. The average values of these parameters for all of the cigarette smoking within the same time span of 0800-1659 and 1700-0159 were also determined.

Results The LF/HF significantly increased $(\mathrm{P}<0.05)$ within 5 minutes from the baseline immediately after smoking. This significant change in LF/HF was observed only at night. Although the interactive effect of the time of day on time course changes was not significant for the $\mathrm{LF} / \mathrm{HF}$ or $\mathrm{HF}$, the reactivity to increase $\mathrm{LF} / \mathrm{HF}$ and decrease HF was more prominent at night.

Conclusions Cigarette smoking significantly increased LF/HF within 5 minutes during ordinary taxi driving. Nighttime smoking seemed to have a more potent acute effect on the cardiac modulation of taxi drivers than in the daytime. The sympathomimetic and parasympatho-withdrawal response of smoking may play an additional role in increasing cardiac risk among taxi drivers.
\end{abstract}

Key terms autonomic nervous activity; cardiovascular disease; circadian rhythm; complex demodulation; parasympathetic activity; smoking; sympathetic activity.

Various epidemiologic studies have revealed that the incidence (1-5) and hospitalization rate $(6,7)$ of cardiovascular disease are high, and taxi-driver patients are characterized by more extensive coronary atherosclerosis in association with a higher prevalence of diabetes mellitus and obesity than nontaxi-driver referents (8). In Japan, a notable number of taxi drivers is involved in karoshi (death from overwork) cases (9). Tokudome (10) also estimated the risk in taxi drivers to be around 16 times that of drivers in the general population by analyzing 20 traffic accident cases caused by sudden and unexpected death during driving. Nevertheless, the mechanisms underlying the increased risk of cardiovascular disease among taxi drivers have not yet been fully investigated. An increase in blood pressure caused by long hours of driving together with various work-related stressors $(4,7,11-15)$, such as dealing with passengers, searching for potential customers along streets and roads, waiting at taxi stands for customers, traffic jams, rain, and highway driving, may contribute substantially to the increased cardiovascular risk. Some neurophysiological mechanisms $(11,15-17)$, including dysfunction of the autonomic nervous system, hyperreactivity of the cardiovascular system, and disturbed circadian rhythms of the endocrine system, may also occasionally result in fatal arrhythmia or chronically accelerated coronary

1 Department of Health and Psychosocial Medicine, Aichi Medical University School of Medicine, Aichi, Japan.

2 Department of Hygiene and Public Health, Osaka Medical College, Osaka, Japan.

3 Faculty of communication studies, Aichi Shukutoku University, Aichi, Japan.

$4 \quad$ Faculty of Nursing, Fujita Health University School of Health Sciences, Fujita, Japan.

5 Department of Internal Medicine and Pathophysiology, Nagoya City University, Graduate School of Medical Sciences, Japan.

Reprint requests to: Dr Fumio Kobayashi, Department of Health and Psychosocial Medicine, Aichi Medical University School of Medicine, 21 Karimata Nagakute-Cho, Aichi, 480-1195, Japan. [E-mail: fumio@ aichi-med-u.ac.jp] 
atherosclerosis. Smoking, which has been reported to be prevalent among taxi drivers $(3,18)$, may have potent acute or chronic effects on these processes. Although smoking is a personal habit, there is cross-sectional evidence that smoking behavior is directly related to the total burden of occupational stressors faced by professional drivers (19). It can therefore contribute to increased cardiovascular risk synergetically with some work-related factors. In our study, we attempted to clarify the acute effects of smoking on autonomic nervous function among taxi drivers under their ordinary work conditions.

\section{Study population and methods}

We asked a company nurse of a taxi company to select about 25 persons from among its approximately 2000 taxi drivers and to recommend them to participate in the study. The selection criteria for the participants were male, aged between 40 and 55 years, working on night duty for at least 1 year, and having no diagnosis of cardiovascular diseases such as coronary heart disease, hypertension, or arrhythmia with no medical treatment, and smoking for more than 1 year. Twenty-three male taxi drivers who agreed to participate in the study after being fully informed of the protocol that had been approved by the Ethical Committee of Aichi Medical University were selected, and written informed consent was obtained from each.

Table 1 shows the overall characteristics of the participants. The mean age was 49.0 (range 42-55) years, and their mean taxi driving experience was 16.7 (range 4-32) years. The participants had smoked a mean of 35.7 (range 10-60) cigarettes per day for 29.3 (range 26-40) years. We analyzed the data recorded between 0800 and 0159 because all of the participants were awake and working during this period. Their health condition was ascertained by reference to health records for the previous 5 years, conducted annually by the taxi company. The age, height, and weight of the participants were obtained from their health check-up record. Information on experience as a driver, the number of cigarettes smoked per day, and years of cigarette smoking was obtained in an interview. The standard workhours were from about 0700 in the morning to 0700 the following day, with possible naps after around 0200, followed by two consecutive nonwork days. The participants were not restricted in terms of their normal daily life activities for the purposes of the study and were instructed to act as they usually would while working.

A Holter electrocardiograph (ECG) (DMC-3253, Nihon Kohden, Japan) was used on each participant. After the participant wore the apparatus in a room at the taxi company, the measurements were started by a time signal (phone no 117) between 0700 and 0750 . The device was removed around 0700 the next morning in the same room. From the waveforms obtained with the Holter ECG, the heartbeat was visually confirmed, and, after arrhythmia and artifacts had been identified and excluded, the R-R interval was determined with an analyzer (DMC-4050, Nihon Kohden). The amplitudes (milliseconds) of the high-frequency (HF) component (central frequency of $0.30 \mathrm{~Hz}$ ) and the low-frequency (LF) component (central frequency of $0.09 \mathrm{~Hz}$ ) were calculated as changes in the R-R interval, and timecourse changes were investigated by a complex demodulation method $(20,21)$. The complex demodulation method (CDM) has been proposed for analyzing the variability of heart rate under nonstationary conditions that provides time-dependent changes in signal amplitude and frequency on a continuous basis, and it may yield insights into short-term changes in autonomic regulation (20). HF was used as the parameter of parasympathetic activity, and the LF to $\mathrm{HF}$ ratio $(\mathrm{LF} / \mathrm{HF})$ was used as the parameter for the dominance of sympathetic activity. The participants were instructed to record the time of their meals in a diary. Data obtained during and up to 30 minutes after meals were omitted. The participants were also instructed to use a specially designed cigarette lighter when they smoked. The voltage generated at the start of ignition was amplified throughout the system, and an event mark on a Holter ECG channel was recorded as $5-\mathrm{Hz}$ rectangular waves for 5 seconds (figure 1). The exact starting time of smoking was identified from the marks. Then the mean LF/HF and HF for 5 minutes just before smoking were calculated as the baseline values, and the means for every 5 minutes after the marks up to 15 minutes were calculated. The series of smoking data that was recorded within 30 minutes of the previous smoking time was omitted from the analysis. We then obtained a series of LF/HF and HF values for each participant for every hour. When there were two marks for a participant in the same hour, a mean value was used as the representative datum. Finally, the average parameters for all of the participants within daytime (0800-1659) and nighttime (1700-0059) were calculated. One participant was excluded because

Table 1. Characteristics of the participants.

\begin{tabular}{lcc}
\hline Characteristic & Mean & SD \\
\hline Age (years) & 49.0 & 3.1 \\
Height $(\mathrm{cm})$ & 163.2 & 5.3 \\
Weight $(\mathrm{kg})$ & 65.8 & 7.1 \\
Body mass index $\left(\mathrm{kg} / \mathrm{m}^{2}\right)$ & 22.9 & 2.6 \\
Experience as a driver (years) & 16.7 & 7.9 \\
Cigarettes smoked per day (N) & 35.7 & 14.7 \\
Cigarette smoking (years) & 29.3 & 3.6 \\
\hline
\end{tabular}




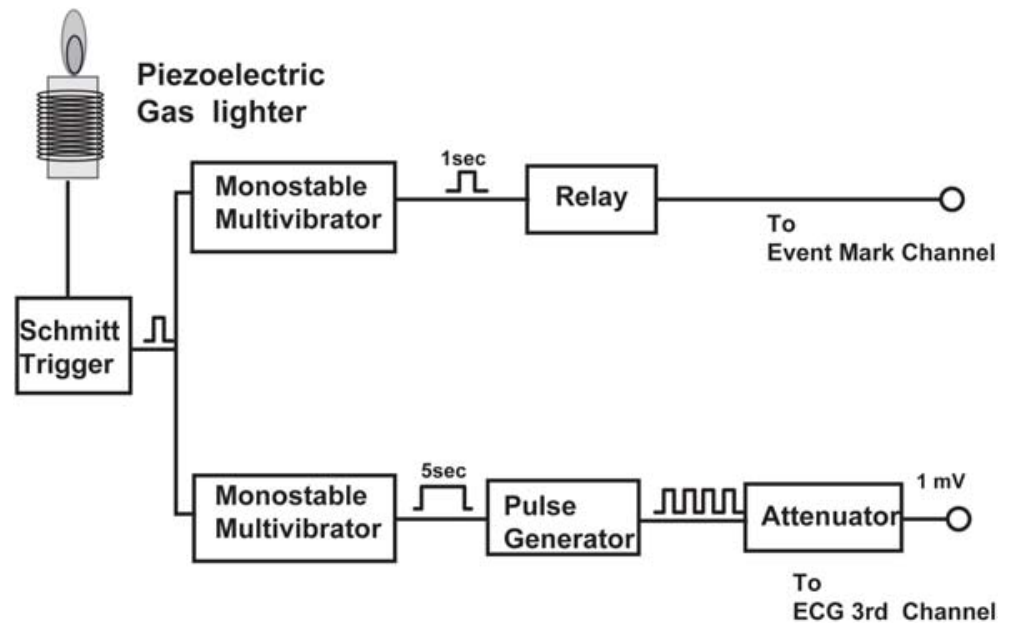

Figure 1. Diagram of a smoking marker circuit. $[E C G=$ electrocardiograph, $\sec =\operatorname{second}(\mathrm{s})]$

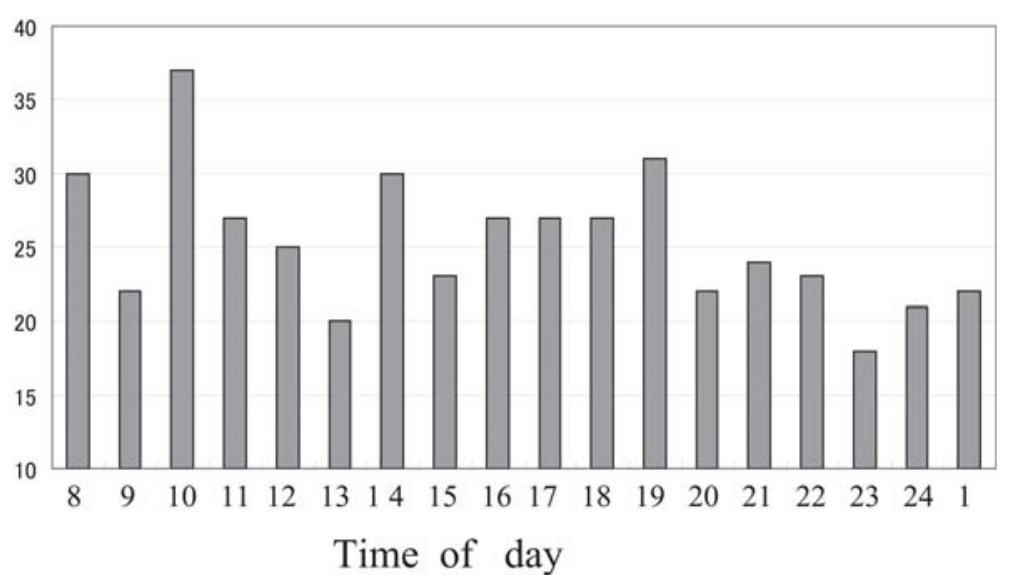

Figure 2. Total number of smoking marks by time of day.
Table 2. Analysis of variance for heart rate variability. ( $d f=d e-$ gree of freedom, LF/HF = ratio of the low-frequency component to the high frequency component of the electrocardiogram, HF = high frequency component of the electrocardiogram)

\begin{tabular}{lccc}
\hline & df & \multicolumn{2}{c}{ F-value } \\
\cline { 2 - 4 } & & LF/HF & HF \\
\cline { 2 - 4 } & & $3.32^{\mathrm{a}}$ & 1.57 \\
Time from the start of smoking (time) & 3 & 0.44 & $13.02^{\mathrm{b}}$ \\
Time span of smoking (time span) & 1 & 0.22 & 0.70 \\
Time $\times$ time span & 3 & & \\
\hline
\end{tabular}

a $P=0.022$

b $P=0.033$.

of a frequent premature ventricular beat, which comprised over $1.0 \%$ of the total heartbeat in his Holter recording. Two participants were also excluded because of their incomplete smoking mark records due to equipment failures. The data from the remaining 20 participants were then used for the analysis. A two (time of day: daytime, nighttime) by four (time from the start of smoking: baseline, 0-5 minutes, 5-10 minutes, 10-15 minutes) analysis of variance (ANOVA) was conducted for LF/HF and HF, followed by Dunnett's two-tailed test. The statistical analysis was performed on a personal computer using the SAS program package, version 8.12 (SAS Institute, Cary, NC, USA), and the critical value was set at a $5 \%$ level of significance.

\section{Results}

Figure 2 shows the total number of marks of smoking by the time of day. From 241 smoking marks in the daytime and 215 at night, we finally obtained 172 and 155 marks, respectively, that were suitable for analysis. Table 2 presents the F-scores of an ANOVA for the time course change in $\mathrm{LF} / \mathrm{HF}$ and $\mathrm{HF}$ (time) and the time of day. For LF/HF, the main effect of time was significant $(\mathrm{P}=0.022)$, but that of the time of day was not. For HF, on the other hand, the main effect of the time of day was significant $(\mathrm{P}=0.033)$, whereas that of time was not. The interaction between time and the time of day was not significant for either $\mathrm{LF} / \mathrm{HF}$ or HF. Table 3 shows the mean $\mathrm{LF} / \mathrm{HF}$ and $\mathrm{HF}$ for the baseline and every 5 minutes after smoking up to 15 minutes by the time of day. The LF/HF increased significantly within 5 minutes 
Table 3. Time course changes in heart rate variability after smoking $(\mathrm{N}=20)$. ( $(\mathrm{FF} / \mathrm{HF}=$ ratio of the low-frequency component to the high frequency component of the electrocardiogram, $\mathrm{HF}=$ high frequency component of the electrocardiogram, day $=0800-1659$, night $=$ 1700-0100)

\begin{tabular}{|c|c|c|c|c|c|c|c|c|c|c|c|c|}
\hline & \multicolumn{6}{|c|}{$\mathrm{LF} / \mathrm{HF}$} & \multicolumn{6}{|c|}{$\mathrm{HF}$} \\
\hline & \multicolumn{2}{|c|}{ Whole day } & \multicolumn{2}{|c|}{ Day } & \multicolumn{2}{|c|}{ Night } & \multicolumn{2}{|c|}{ Whole day } & \multicolumn{2}{|c|}{ Day } & \multicolumn{2}{|c|}{ Night } \\
\hline & Mean & SD & Mean & SD & Mean & SD & Mean & SD & Mean & SD & Mean & SD \\
\hline Baseline & 3.85 & 0.50 & 3.88 & 0.51 & 3.83 & 0.53 & 14.49 & 1.37 & 14.07 & 1.44 & 14.76 & 1.39 \\
\hline$\sim 5$ minutes & 4.30 & $0.54^{a}$ & 4.28 & 0.54 & 4.32 & $0.54^{a}$ & 14.07 & 1.25 & 13.84 & 1.32 & 14.24 & 1.25 \\
\hline 10 minutes & 4.12 & 0.54 & 4.00 & 0.56 & 4.20 & 0.56 & 14.49 & 1.41 & 14.34 & 1.47 & 14.65 & 1.45 \\
\hline$\sim 15$ minutes & 3.83 & 0.48 & 3.75 & 0.48 & 3.89 & 0.51 & 14.37 & 1.33 & 13.85 & 1.30 & 14.74 & 1.43 \\
\hline
\end{tabular}

a $P<0.05$, changes from the baseline values, Dunnett's test.
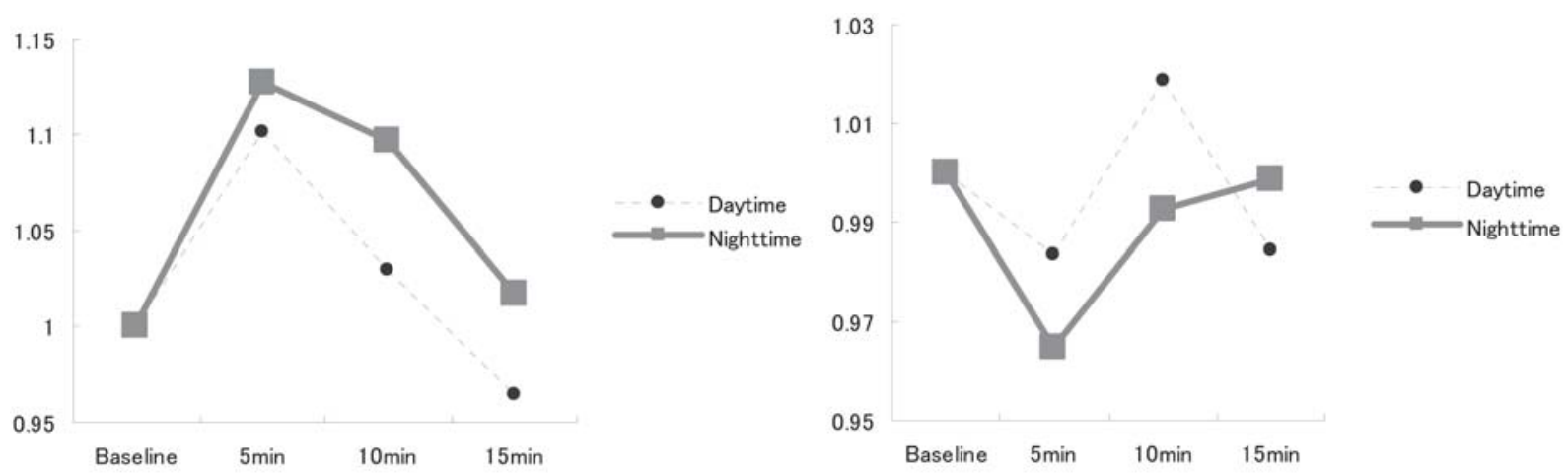

Figure 3. Comparison of the degree of change from the baseline level between daytime and nighttime.

from the baseline immediately after smoking $(\mathrm{P}<0.05$, Dunnet's test) and returned to the baseline level within 15 minutes. This significant change in LF/HF was observed only at night. Although the time course change in HF did not reach a significant level, it showed a tendency to decrease within 5 minutes immediately after a participant smoked and to recover towards the baseline level for 15 minutes. During the day, the HF decreased within 5 minutes and then showed a remarkable increase for up to 10 minutes. This pattern of change differed from that of nighttime. In figure 3 , the degree of change in $\mathrm{LF} / \mathrm{HF}$ and $\mathrm{HF}$ is compared between the daytime and nighttime by presenting the ratio of the parameters for each time section divided by the baseline values. For $\mathrm{LF} / \mathrm{HF}$, the ratio at night proved to be higher throughout the measurements. For HF, a prominent decrease was observed only at night.

\section{Discussion}

We used LF/HF and HF as indices of cardiac autonomic nervous function. There is substantial evidence that HF components are a reliable index of vagus-mediated parasympathetic activity, but it remains controversial as to whether changes in LF/HF are a marker of sympathetic activity $(22,23)$. Some investigators, however, consider the LF/HF to mirror the sympathovagal balance $(23,24)$. This study did not regard LF/HF as a parameter of separate cardiac sympathetic nervous activity, but rather as an index of sympathetic predominance determined by sympathetic activation or parasympathetic withdrawal.

Time-course changes in these two parameters were investigated with the use of the CDM. Hayano, in a simulation study (20), examined the reliability and validity of the CDM and showed that it has sufficient frequency resolution to measure low and high frequency amplitudes separately, with a time resolution of less than 15 seconds, and that it is robust in relation to changes in the frequency of the components. In a field study such as ours, CDM is therefore more suitable for assessing acute changes in cardiac autonomic parameters in occupational psychophysiological studies than the Fourier transform, which needs much longer stationary periods (25).

We found a significant acute increase in LF/HF after smoking. While the decreasing tendency of HF did not reach a significant level, these findings are consistent with the results of previous studies suggesting either acute sympathetic activation or parasympathetic withdrawal or both as a result of smoking (26-28). These acute effects of smoking have also been reported in smoking cessation intervention $(28,29)$, with a rapid increase in heart rate variability appearing as quickly as 1 day after smoking cessation. As it is well established 
that plasma catecholamine levels increase within 1 minute after a person smokes $(30,31)$, the short-term changes in LF/HF and HF after smoking should depend on an increased release or a reduced clearance of catecholamines at the neuroeffector junctions (32). Therefore, it is highly probable that the parameter changes observed within 15 minutes after smoking in our study are a consequence of cigarette smoking.

Although we could not find any statistically significant effects of the time of day on the time-course changes in cardiac autonomic parameters after smoking (table 2), LF/HF showed a significant increase immediately after smoking only at night (table 3 ), while sympathomimetic or parasympatho-withdrawal effects seemed to be more prominent at night than during the day (figure 3). These data suggest that smoking at night has a more potent acute effect on the cardiac modulation of taxi drivers than during the day. Previous studies have confirmed the presence of a circadian rhythm with the prevalence of sympathetic activity during the day and a marked increase in parasympathetic activity during the night (14, 33-35). At night, smoking taxi drivers are therefore assumed to have their own circadian rhythms, which originally decrease sympathetic activities and increase parasympathetic ones, disturbed by the effects of smoking. These conflicting effects of smoking on circadian rhythm may contribute to some extent to the increase the physiological burden $(14,36)$ of taxi drivers.

In an experimental study, LF/HF more than doubled towards the end of a 4-hour drive when compared with the LF/HF of the earlier period, and there was a significant linear increase in $0.1 \mathrm{~Hz}$ heart rate variability as a function of the distance driven (37). In addition, the urinary excretion of norepinephrine generally increased at the end of the workday and during driving in fog (38). From these results, the effect of work-related factors such as long taxi driving or long workhours (11) on cardiac autonomic modulation may be increased by smoking, especially during nighttime work.

Enhanced sympathetic or decreased vagal modulation of cardiac function has been associated with an increased risk of coronary heart disease and mortality (39, 40) and angiographic progression of coronary atherosclerosis (41), as well as arrhythmia and sudden cardiac death (25). Thus smoking at work, the prevalence of which is high among professional drivers $(3,18)$, especially among Japanese taxi drivers $(42,43)$, may play an additional role in the increased risk of cardiovascular morbidity and mortality via unfavorable effects on cardiac autonomic nervous balance in taxi drivers.

\section{Clinical implications}

To eliminate the confounding effects of aging, gender, and ongoing medical treatment, we selected only untreated middle-aged male taxi drivers for our study. In addition, their average duration of work experience as a taxi driver was over 16 years, and they were therefore well experienced and adapted to their work. The study therefore might show the effect of smoking on a highly selected healthy group. Acute sympathetic activation through smoking at night might have a much more potent effect on the progression of coronary atherosclerosis or even be likely to trigger cardiovascular events in taxi drivers who are less experienced, aged, with some coronary risk factors or diseases such as diabetes, hypertension, arrhythmia, or other ischemic coronary heart diseases.

\section{Limitations}

We did not attempt to standardize the smoking behavior of the participants, for example, how frequently should they smoke per minute, when to stop smoking, and so on. Therefore, the smoking behavior during the day may have had carry-over effects on the cardiac response at night. We could not control many other factors affecting cardiac autonomic modulation, and data during and up to 30 minutes after meals of the participants were omitted because of the obvious sympathoactivating effects. Postures, physical activities, caffeine intake, or temperatures, for instance, may have also influenced the cardiac autonomic balance. In addition, in their everyday work, Japanese taxi drivers are exposed to the second-hand cigarette smoke of their smoking passengers. Acute short-term passive smoke exposure has been shown to alter cardiac autonomic function (32) or elicit a modest increase in muscle sympathetic nerve activity in healthy nonsmokers (44). The relationship between circadian rhythm and smoking effects was discussed only on the basis of the data between 0800 and 0100 by comparing the two roughly divided times because of the small number of participants. Figure 3 shows that HF increased 5-10 minutes after smoking only in the daytime. Some vagal compensatory mechanisms are thought to be possible (26), but this possibility has not yet been fully investigated. The time course change of the parameters may, therefore, be somewhat unclear due to such a variety of factors. A more precise acute smoking effect on cardiac autonomous modulation after control for the aforementioned factors should be clarified in well-designed experimental studies in the future.

\section{Concluding remarks}

Cigarette smoking significantly increased LF/HF within 5 minutes during ordinary taxi driving. Nighttime smoking seemed to have a more potent acute effect on the cardiac modulation of taxi drivers than daytime 
smoking. The sympathomimetic and parasympathowithdrawal response of smoking may play an additional role in increasing cardiac risk among taxi drivers.

\section{Acknowledgments}

This study was supported by grants-in-aid for scientific research from the Japan Ministry of Education, Culture, Sports, Science and Technology.

\section{References}

1. Hattori M. Long-term follow-up of ischemic heart disease in taxi drivers and the influence of taxi driving on their cardiovascular and autonomic nerve functions I: occupational factors related to ischemic changes in post-exercise electrocardiograms and a cohort study on the prediction of incidence of ischemic heart disease. Sci Labour 1989;65(11):580-9.

2. Gustavsson P, Alfledsson L, Brunnberg H, Hammar N, Jakobsson $\mathrm{R}$, Reuterwall C, et al. Myocardial infarction among male bus, taxi, and lorry drivers in middle Sweden. Occup Environ Med 1996;53:235-40.

3. Rosengren A, Anderson K, Wilhelmsen L. Risk of coronary heart disease in middle-aged male bus and tram drivers compared to men in other occupations: a prospective study. Int J Epidemiol 1991;20(1):82-7.

4. Bigert C, Gustavsson P, Hallqvist J, Hogstedt C, Lewne M, Plato N, et al. Myocardial infarction among professional drivers. Epidemiology 2003;14(3):333-9.

5. Hammar N, Alfredsson L, Smedberg M, Ahlbom A. Differences in the incidence of myocardial infarction among occupational groups. Scand J Work Environ Health. 1992; 18(3):178-85.

6. Tüchsen F, Bach E, Marmot M. Occupation and hospitalization with ischaemic heart diseases: a new nationwide surveillance system based on hospital admissions. Int J Epidemiol 1992;21(3):450-9.

7. Hannerz H, Tüchsen F. Hospital admissions among male drivers in Denmark. Occup Environ Med 2001;58(4):253-60.

8. Kurosaka K, Daida H, Muto T, Watanabe Y, Kawai S, Yamaguchi H. Characteristics of coronary heart disease in Japanese taxi drivers as determined by coronary angiographic analyses. Ind Health 2000;38(1):15-23.

9. Uehata T. A study on occupational stress factors triggering cardiac and cerebrovascular attacks. Rodo Kagaku 1982; 58(6);277-293.

10. Tokudome S. Cases of traffic accident of sudden and unexpected death while driving. Baishou Igaku 1988;7:77-82.

11. Emdad R, Belkić K, Theorell T, Cizinsky S, Savić C, Olsson $\mathrm{K}$. Work environment, neurophysiologic and psychophysiologic models among professional drivers with and without cardiovascular disease: seeking an integrative neurocardiologic approach. Stress Med 1997;13:7-21.

12. Belkić K, Ercegovać D, Savić C, Panić B, Djordjević M, Savić S. EEG arousal and cardiovascular reactivity in professional drivers: the glare pressor test. Eur Heart J 1992;13:304-9.

13. Belkić K, Emdad R, Theorell T. Occupational profile and cardiac risk: possible mechanisms and implications for pro- fessional drivers. Int J Occup Med Environ Health 1998; 11(1):37-57.

14. Kobayashi F, Watanabe T, Watanabe M, Akamatsu Y, Tomita $\mathrm{T}$, Nakane $\mathrm{T}$, et al. Blood pressure and heart rate variability in taxi drivers on long duty schedules. J Occup Health 2002;44:214-20.

15. Hattori M. Long-term follow-up of ischemic heart disease in taxi drivers and the influence of taxi driving on their cardiovascular and autonomic nerve functions, II: the influence of taxi driving on cardiovascular and autonomic functions. Sci Labour 1989;65:651-8.

16. Belkić K, Savić C, Theorell T, Rakić L, Ercegovac D, Djordjević M. Mechanisms of cardiac risk among professional drivers [review]. Scand J Work Environ Health 1994;20:73-86.

17. Maehara N, Sasaki T, Li Q, Sawa M, Mori K, Hanaoka T, et al. The incidence of ventricular premature contractions on a workday, the first day-off after a night shift and a holiday of three taxi drivers engaged in mid-night driving. Sci Labour 1996;72:396-412.

18. Holme I, Helgeland A, Hjermann I, Leren P, Lund-Larsen PG. Coronary risk factors in various occupational groups: the Oslo study. Br J Prev Soc Med 1977;31:96-100.

19. Emdad R, Belkić K, Theorell T, Cizinsky S. What prevents professional drivers from following physicians' cardiologic advice? Psychother Psychosom 1998;67(4-5):226-40.

20. Hayano J, Taylor JA, Mukai S, Hori R, Asakawa T, Yokoyama K, et al. Continuous assessment of hemodynamic control by complex demodulation of cardiovascular variability. Am J Physiol 1993;246:H1229-38.

21. Shin SJ, Tapp WN, Reisman SS, Natelson BH. Assessment of autonomic regulation of heart rate variability by the method of complex demodulation. IEEE Trans Biomed Eng 1989;36:274-83.

22. Eckberg DL. Sympathovagal balance: a critical appraisal. Circulation 1997;96(9):3224-32.

23. Task Force of the European Society of Cardiology and the North American Society of Pacing and Electrophysiology. Heart rate variability: standards of measurement, physiological interpretation and clinical use. Circulation 1996; 93(5):1043-65.

24. Nabors-Oberg RE, Niaura RS, Sollers JJ 3rd, Thayer JF. The effects of controlled smoking on heart period variability. IEEE Eng Med Biol Mag 2002;21(4):65-70.

25. Wilhelm FH, Grossman P, Roth WT. Assessment of heart rate variability under non-stationary conditions: complex demodulation vs. spectral analysis. Psychophysiology 1997;34 suppl 1:S96.

26. Hayano J, Yamada M, Sakakibara Y, Fujinami T, Yokoyama $\mathrm{K}$, Watanabe Y, et al. Short- and long-term effects of cigarette smoking on heart rate variability. Am J Cardiol 1990; 65(1):84-8.

27. Narkiewicz K, van de Borne PJ, Hausberg M, Cooley RL, Winniford MD, Davison DE, et al. Cigarette smoking increases sympathetic outflow in humans. Circulation 1998; 98(6):528-34.

28. Minami J, Ishimitsu T, Matsuoka H. Effects of smoking cessation on blood pressure and heart rate variability in habitual smokers. Hypertension 1999;33(1 Pt 2):586-90.

29. Yotsukura M, Koide Y, Fujii K, Tomono Y, Katayama A, Ando $\mathrm{H}$, et al. Heart rate variability during the first month of smoking cessation. Am Heart J 1998; 135(6 Pt 1):1004-9.

30. Cryer PE, Haymond MW, Santiago JV, Shah SD. Norepinephrine and epinephrine release and adrenergic mediation of smoking-associated hemodynamic and metabolic events. N 
Engl J Med 1976;295(11):573-7.

31. Hill P, Wynder EL. Smoking and cardiovascular disease: effect of nicotine on the serum epinephrine and corticoids. Am Heart J 1974;87(4):491-6.

32. Grassi G, Seravalle G, Calhoun DA, Bolla GB, Giannattasio $\mathrm{C}$, Marabini M, et al. Mechanisms responsible for sympathetic activation by cigarette smoking in humans. Circulation 1994;90(1):248-53.

33. Sapoznikov D, Luria MH, Mahler Y, Gotsman MS. Day vs night ECG and heart rate variability patterns in patients without obvious heart disease. J Electrocardiol 1992;25(3):17584.

34. Huikuri HV, Kessler KM, Terracall E, Castellanos A, Linnaluoto MK, Myerburg RJ. Reproducibility and circadian rhythm of heart rate variability in healthy subjects. Am J Cardiol 1990;65(5):391-3.

35. Guo YF, Stein PK. Circadian rhythm in the cardiovascular system: considerations in non-invasive electrophysiology. Card Electrophysiol Rev 2002;6(3):267-72.

36. Sato S, Taoda K, Kawamura M, Wakaba K, Fukuchi Y, Nishiyama K. Heart rate variability during long truck driving work. J Hum Ergol (Tokyo) 2001;30(1-2):235-40.

37. Egelund N. Spectral analysis of heart rate variability as an indicator of driver fatigue. Ergonomics 1982;25(7):663-72.

38. Vivoli G, Bergomi M, Rovesti S, Carrozzi G, Vezzosi A. Biochemical and haemodynamic indicators of stress in truck drivers. Ergonomics 1993;36(9):1089-97.

39. Dekker JM, Crow RS, Folsom AR, Hannan PJ, Liao D,
Swenne CA, et al. Low heart rate variability in a 2-minute rhythm strip predicts risk of coronary heart disease and mortality from several causes: the ARIC Study. Circulation 2000;102(11):1239-44.

40. Mäkikallio TH, Hoiber S, Kober L, Torp-Pedersen C, Peng $\mathrm{CK}$, Goldberger AL, et al. Fractal analysis of heart rate dynamics as a predictor of mortality in patients with depressed left ventricular function after acute myocardial infarction. Am J Cardiol 1999;83(6):836-9.

41. Huikuri HV, Jokinen V, Syvänne M, Nieminen MS, Airaksinen KE, Ikäheimo MJ, et al. Heart rate variability and progression of coronary atherosclerosis. Arterioscler Thromb Vasc Biol 1999;19(8):1979-85.

42. Ueda T, Hashimoto M, Yasui I, Sunaga M, Higashida T, Hara I. A questionnaire study on health of taxi drivers-relation to work conditions and daily life-. Jpn J Ind Health 1989; $31: 162-75$.

43. Kishi R, Fukuoka M, Watanabe T, Fujimura N, Yoshioka N, Yamazaki Y, et al. A survey on health problems among taxi drivers and public transport service workers. J North Occup Health 1991;38:50-7.

44. Hausberg M, Mark AL, Winniford MD, Brown RE, Somers VK. Sympathetic and vascular effects of short-term passive smoke exposure in healthy nonsmokers. Circulation 1997;96(1):282-7.

Received for publication: 12 November 2004 